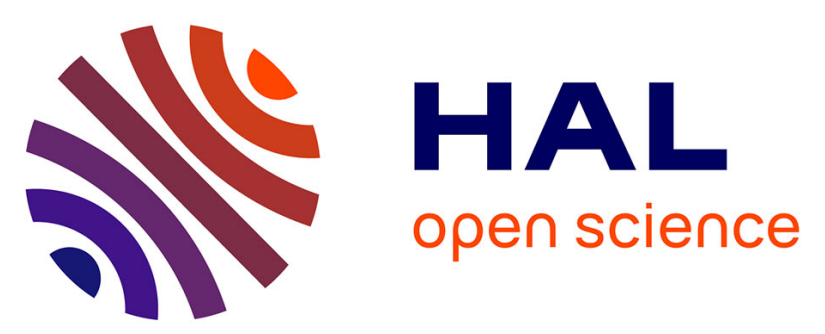

\title{
Influence of the elastic deformations on the form factor of polyhedral nanocrystals: the illustrative example of the pseudomorphic inclusion
}

\author{
Bernard Croset
}

\section{- To cite this version:}

Bernard Croset. Influence of the elastic deformations on the form factor of polyhedral nanocrystals: the illustrative example of the pseudomorphic inclusion. Journal of Applied Crystallography, 2019, 52 (2), pp.387-393. 10.1107/S1600576719002553 . hal-02083409

\section{HAL Id: hal-02083409 \\ https: / hal.sorbonne-universite.fr/hal-02083409}

Submitted on 3 Apr 2019

HAL is a multi-disciplinary open access archive for the deposit and dissemination of scientific research documents, whether they are published or not. The documents may come from teaching and research institutions in France or abroad, or from public or private research centers.
L'archive ouverte pluridisciplinaire HAL, est destinée au dépôt et à la diffusion de documents scientifiques de niveau recherche, publiés ou non, émanant des établissements d'enseignement et de recherche français ou étrangers, des laboratoires publics ou privés. 


\section{Influence of the elastic deformations on the form factor of polyhedral nanocrystals: the illustrative example of the pseudomorphic inclusion}

\section{Bernard Croset}

J. Appl. Cryst. (2019). 52, 387-393

\section{IUCr Journals CRYSTALLOGRAPHY JOURNALS ONLINE}

Copyright (C) International Union of Crystallography

Author(s) of this article may load this reprint on their own web site or institutional repository provided that this cover page is retained. Republication of this article or its storage in electronic databases other than as specified above is not permitted without prior permission in writing from the IUCr.

For further information see http://journals.iucr.org/services/authorrights.html 
JOURNAL OF

APPLIED

CRYSTALLOGRAPHY

ISSN 1600-5767

Received 7 January 2019

Accepted 19 February 2019

Edited by V. Holý, Charles University, Prague, Czech Republic and CEITEC at Masaryk

University, Brno, Czech Republic

Keywords: coherent scattering; form factor; elasticity; elastic deformations; X-rays.

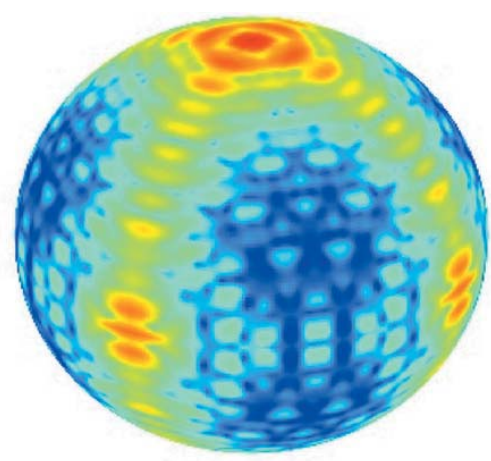

C 2019 International Union of Crystallography

\section{Influence of the elastic deformations on the form factor of polyhedral nanocrystals: the illustrative example of the pseudomorphic inclusion}

\author{
Bernard Croset* \\ Sorbonne Université, CNRS-UMR 7588, Institut des NanoSciences de Paris, F-75005, Paris, France. *Correspondence \\ e-mail: bernard.croset@insp.jussieu.fr
}

Using an analytically tractable example, the pseudomorphic inclusion, this article examines the influence of elastic deformations on the form factor of polyhedral nanocrystals. A control parameter, the total amplitude of the variation of the complex density phase, is identified and it is shown that for low enough deformations the characteristic asymptotic behaviours as a function of the scattering vector associated with the polyhedral crystal shape are preserved, leading to a strong contrast in the dependence of the form factor on orientation. Using the sections method, it is explained why these results can be generalized to more realistic elastic situations.

\section{Introduction}

In a previous article, we have established a compact formula for the form factor of polyhedral crystals (Croset, 2017). We have shown that the amplitude form factor presents an asymptotic behaviour in $q^{-1}$ when the scattering vector, $\mathbf{q}$, is normal to a face and an asymptotic behaviour in $q^{-2}$ when the scattering vector is perpendicular to an edge, the generic asymptotic behaviour being in $q^{-3}$. Therefore, the angular dependence of the form factor exhibits marked contrast for large enough $q$. The sections method developed in a subsequent article (Croset, 2018) showed that these asymptotic behaviours and this contrast are intrinsically linked to the finite character of the crystal which leads to discontinuities of the density at the crystal surface.

Moreover, coherent diffraction experiments have shown that elastic deformations modify the scattering pattern around each Bragg peak of a diffracting nanocrystal (Pfeifer et al., 2006; Diaz et al., 2010; Xiong et al., 2014; Chamard et al., 2015; Hruszkewycz et al., 2017; Favre-Nicolin et al., 2018; Hill et al., 2018; Shin et al., 2018). A standard approximation shows that, in the calculation of the form factor, the real density, $\rho(\mathbf{r})$, must be replaced by a complex one of which the modulus is the real density and the phase is the scalar product, $-\mathbf{Q} \cdot \mathbf{u}(\mathbf{r})$, between $\mathbf{Q}$, the Bragg vector, and $\mathbf{u}(\mathbf{r})$, the displacement. Two cases must be distinguished: the case of plastic deformations and the case of elastic deformations. In the first case - dislocations, grain boundaries etc. - the displacements present discontinuities which lead to discontinuities of the complex density inside the crystal. Therefore, important variations of the asymptotic behaviour of the form factor are expected in such a case. In the second case, when compared with the undeformed case, because of variations of its phase, the density is no longer constant in the crystal but its discontinuities and singularities still occur solely at the crystal surface. 
We may expect that, in such a case, the modifications of the form factor asymptotic behaviour are more subtle. A question emerges: is the contrast in the form factor dependence on $q$ orientation modified by elastic deformations? This article is devoted to answering this question.

The study of the influence of elastic deformations on the form factor implies the calculation of the elastic displacements. In spite of the apparent simplicity of the constitutive equations of the theory of continuous media linear elasticity, the calculation of displacements is rarely analytical and necessitates numerical approaches (molecular dynamics or the finite element method). In this topic, pseudomorphic inclusions, i.e. crystalline precipitates exhibiting the same crystalline parameters as their crystalline host matrix, play a special role as it has been known since the seminal work of Eshelby (1957) that the elastic displacements of a strained pseudomorphic inclusion can be calculated analytically. We will study this case as an illustrative example of the behaviour of the form factor in the presence of elastic deformations. In the first part, we will describe the analytical calculation of the elastic displacements of a cubic pseudomorphic inclusion. Then, in the second part, we will discuss the results of the numerical computation of the form factor for various orientations of the stress and of the Bragg vector.

\section{Calculations of the elastic displacements in a cubic pseudomorphic inclusion}

In the absence of body forces, the constitutive equations of linear elasticity are written (Lifshitz \& Landau, 1995)

$$
\begin{gathered}
\bar{\sigma}=\overline{\bar{C}} \cdot \bar{\varepsilon}, \\
\varepsilon_{i j}=1 / 2\left(\partial_{i} u_{j}+\partial_{j} u_{i}\right), \\
\operatorname{div} \bar{\sigma}=\mathbf{0}, \\
\mathbf{F}=\left(\bar{\sigma}_{\text {out }}-\bar{\sigma}_{\text {in }}\right) \cdot \mathbf{n},
\end{gathered}
$$

where $\bar{\sigma}$ is the stress tensor, $\bar{\varepsilon}$ is the strain tensor, $\overline{\bar{C}}$ is the stiffness tensor and $\mathbf{n}$ is the normal to the surface on which forces $\mathbf{F}$ are applied. Following Eshelby (1957), we will search for the displacement field associated with a pseudomorphic inclusion in an infinite crystal. A first approximation is to take the same stiffness tensor in the inclusion and in the crystal. A second approximation is to take a stiffness tensor corresponding to the isotropic case; it is therefore given by the knowledge of two elastic constants, the Young modulus, E, and the Poisson coefficient, $v$. These two approximations allow one to perform analytical calculations. Because of different lattice constants between the pseudomorphic inclusion and the surrounding crystal, the pseudomorphic inclusion is strained. Let us denote by $\bar{\varepsilon}_{0}$ the uniform strain due to this difference in lattice constants. The strain and stress fields, $\bar{\varepsilon}_{1}(\mathbf{r})$ and $\bar{\sigma}_{1}(\mathbf{r})$, which are equal to $\overline{0}$ outside the inclusion and to $\bar{\varepsilon}_{0}$ and $\bar{\sigma}_{0}=\overline{\bar{C}} \cdot \bar{\varepsilon}_{0}$ inside the inclusion, satisfy equations (1), (2) and (3). However, these fields do not satisfy equation (4) since the discontinuity of $\bar{\sigma}_{1}$ is unbalanced at the inclusion surface.
Let us consider the surface forces, $\mathbf{F}_{1}$, defined by

$$
\mathbf{F}_{1}=-\left(\bar{\sigma}_{1, \text { out }}-\bar{\sigma}_{1, \text { in }}\right) \cdot \mathbf{n}=\bar{\sigma}_{0} \cdot \mathbf{n} .
$$

Using the linearity of the constitutive equations, if we are able to find two fields $\bar{\varepsilon}_{2}(\mathbf{r})$ and $\bar{\sigma}_{2}(\mathbf{r})$ which satisfy equations (1), (2) and (3) and satisfy equation (4) with $\mathbf{F}=\mathbf{F}_{1}$, the sum of the fields 1 and 2 will answer our problem since it presents no stress discontinuities at the inclusion surface. For $\sigma_{2}$, equation (4) can be written

$$
\left(\bar{\sigma}_{2, \text { out }}-\bar{\sigma}_{2, \text { in }}\right) \cdot \mathbf{n}=\mathbf{F}_{1}=-\left(\bar{\sigma}_{1, \text { out }}-\bar{\sigma}_{1, \text { in }}\right) \cdot \mathbf{n},
$$

which leads to

$$
\left[\left(\bar{\sigma}_{1, \text { out }}+\bar{\sigma}_{2, \text { out }}\right)-\left(\bar{\sigma}_{1, \text { in }}+\bar{\sigma}_{2, \text { in }}\right)\right] \cdot \mathbf{n}=\mathbf{0} .
$$

For an infinite elastic isotropic medium, the Green function, i.e. the displacement field due to a point force, $\mathbf{F} \delta_{r^{\prime}}$, is known. We have

$$
\mathbf{u}(\mathbf{r})=\frac{1+v}{8 \pi E(1-v)} \frac{(3-4 v) \mathbf{F}+\mathbf{l}\left(\mathbf{r}-\mathbf{r}^{\prime}\right)\left[\mathbf{F} \cdot \mathbf{l}\left(\mathbf{r}-\mathbf{r}^{\prime}\right)\right]}{\left|\mathbf{r}-\mathbf{r}^{\prime}\right|},
$$

where $\mathbf{l}(\mathbf{r})=\mathbf{r} / r$.

Thanks to our two approximations, equality of the stiffness coefficients in the inclusion and the surrounding crystal and isotropy of its stiffness tensor, we can use this Green function to calculate $\mathbf{u}_{2}(\mathbf{r})$. We obtain

$$
\mathbf{u}_{2}(\mathbf{r})=\iint_{S^{\prime}} \bar{H}\left(\mathbf{r}, \mathbf{r}^{\prime}\right) \cdot \mathbf{n}\left(\mathbf{r}^{\prime}\right) \mathrm{d}^{2} S^{\prime}
$$

with $\bar{H}$ given by

$$
\bar{H}\left(\mathbf{r}, \mathbf{r}^{\prime}\right)=\frac{1+v}{8 \pi E(1-v)} \frac{(3-4 v) \bar{\sigma}_{0}+\mathbf{l}\left(\mathbf{r}-\mathbf{r}^{\prime}\right) \otimes\left[\bar{\sigma}_{0} \cdot \mathbf{l}\left(\mathbf{r}-\mathbf{r}^{\prime}\right)\right]}{\left|\mathbf{r}-\mathbf{r}^{\prime}\right|},
$$

where $\otimes$ stands for the tensorial product between two vectors. Using the Gauss theorem, we can write

$$
\mathbf{u}_{2}(\mathbf{r})=\iiint_{V^{\prime}} \bar{H}\left(\mathbf{r}, \mathbf{r}^{\prime}\right) \cdot \operatorname{div} \mathrm{d}^{3} v^{\prime}=\iiint_{V^{\prime}} \frac{\mathbf{h}\left[\mathbf{l}\left(\mathbf{r}-\mathbf{r}^{\prime}\right)\right]}{\left|\mathbf{r}-\mathbf{r}^{\prime}\right|^{2}} \mathrm{~d}^{3} v^{\prime},
$$

where each component of $\mathbf{h}(\mathbf{l})$ is an odd polynomial of degree three in the components of $\mathbf{I}$, of which the coefficients are linear combinations of the $\bar{\sigma}_{0}$ components. Since $\overline{\bar{C}}$ is proportional to $E$, the relation between $\mathbf{u}_{2}$ and $\bar{\varepsilon}_{0}$ is linear with coefficients only depending on $v$ and on the geometry of the inclusion but is independent of $E$. The choice of a cuboid for the inclusion shape allows us to keep the analyticity of the calculation one step further than equation (11) since real antiderivatives can be used to perform each step of the triple summation of the different terms of $h$. Finally we obtain

$$
\mathbf{u}_{2}(\mathbf{r})=A_{\mathbf{r}}\left(\bar{\sigma}_{0}\right)
$$

where $A_{\mathbf{r}}$ is a linear operator acting on $\bar{\sigma}_{0}$ of which all the elements can be analytically computed.

Before leaving this section, an important remark must be made. Dimensional analysis allows us to show that the 
abandonment of our two approximations will keep the general form of $\bar{H}\left(\mathbf{r}, \mathbf{r}^{\prime}\right)$, which will be written

$$
\bar{H}\left(\mathbf{r}, \mathbf{r}^{\prime}\right)=\frac{\overline{\bar{D}}\left[\mathbf{l}\left(\mathbf{r}-\mathbf{r}^{\prime}\right)\right]}{\left|\mathbf{r}-\mathbf{r}^{\prime}\right|} \cdot \bar{\sigma}_{0} .
$$

This allows us to understand why the deformation landscape based on these two approximations is generally admitted to be good. Moreover, careful examination of equation (11) shows that, for a given $\bar{\varepsilon}_{0}$ and $\bar{\sigma}_{0}$, a homothetic transformation of the crystal given by $r \rightarrow s=r e$ and $V^{\prime} \rightarrow W^{\prime}$ leads to a displacement field obeying the transformation $u_{2}(r) \rightarrow e u_{2}(s / e)$.

\section{Analysis of the numerically computed form factor}

The analytical calculations of the displacement field allow us to perform by $3 \mathrm{D}$ fast Fourier transform the numerical computation of the form factor around a given Bragg peak using the relation

$$
\begin{aligned}
f(\mathbf{q}) & =\iiint_{V} \exp \left\{-i \mathbf{s} \cdot\left[\mathbf{r}+\mathbf{u}_{2}(\mathbf{r})\right]\right\} \mathrm{d}^{3} v \\
& \simeq \iiint_{V} \exp \left[-i \mathbf{Q} \cdot \mathbf{u}_{2}(\mathbf{r})\right] \exp (-i \mathbf{q} \cdot \mathbf{r}) \mathrm{d}^{3} v \\
& =\iiint_{V} \exp \left[-i \phi\left(r ; \mathbf{Q}, \sigma_{0}\right)\right] \exp (-i \mathbf{q} \cdot \mathbf{r}) \mathrm{d}^{3} v
\end{aligned}
$$

where $\mathbf{s}$ is the scattering vector, $\mathbf{Q}$ is the Bragg vector and $\mathbf{q}=$ $\mathbf{s}-\mathbf{Q}$ is the 'reduced' scattering vector. It is clear that the dependence of the density phase, $\phi$, on $\mathbf{Q}$ and $\bar{\sigma}_{0}$ will play an essential role. $\phi$ depends linearly on $\mathbf{Q}$ and we have seen in the previous section that $\mathbf{u}_{2}$ depends linearly on $\sigma_{0}$. Therefore, the shape of the phase landscape is controlled by the directions of $\mathbf{Q}$ and $\bar{\sigma}_{0}$ while its contrast is controlled by the product of their two amplitudes. Consequently, the study of the form factor of a highly deformed crystal around a low-index Bragg peak is equivalent to the study of a weakly deformed crystal around a high-index Bragg peak. In the following, we will discuss the form factor behaviour for different directions of $\mathbf{Q}$ and $\bar{\sigma}_{0}$ using for the Poisson coefficient, $v$, the standard value of 0.3 . In order to keep the origin of the form factor constant, the displacements used for the calculation of the complex density are corrected by a linear part corresponding to the average of the deformation on the crystal. $\Delta \phi$, the difference between the maximum and the minimum of the phase, will be used as a control parameter. We will see that $2 \pi$ appears to be a central value for $\Delta \phi$. From a scattering point of view, it is clear that $f(\mathbf{q})$ will be quite similar to the undeformed crystal form factor for $\Delta \phi \ll 2 \pi$ while deformations will play a dominant role for $\Delta \phi \gg 2 \pi$. From a physical point of view, for low-index Bragg peaks a dislocation corresponds to $\Delta \phi=2 \pi$ (Stroh, 1958; Ting, (b)
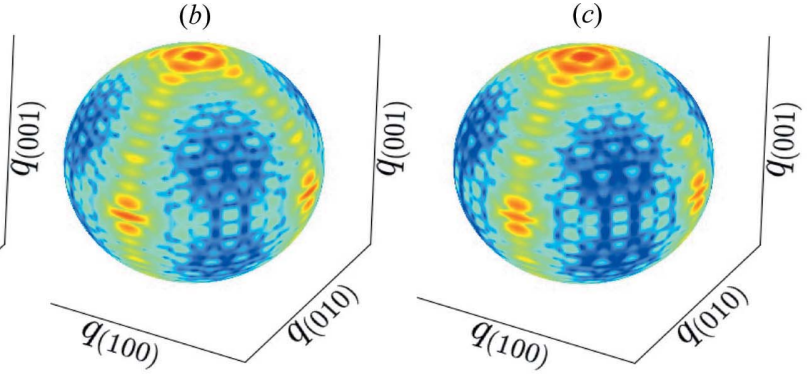

(e)
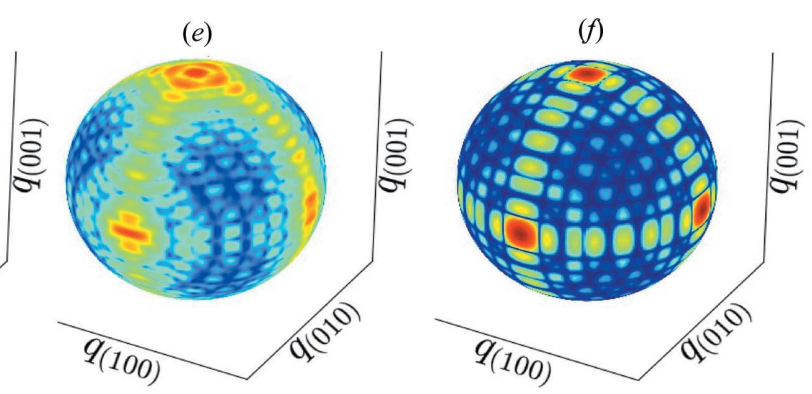

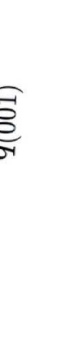

Orientation dependence of the amplitude form factor of a cubic inclusion of size $L$ for $q L=20$ and for $\Delta \phi=2 \pi$. The colour scale is logarithmic and common to all the images. The form factor amplitude, $f$, is normalized by the cube volume, $V$. The $\mathbf{Q}$ direction is (001). The shear stresses are null for all the images. $(a)\left(\sigma_{x x}, \sigma_{y y}, \sigma_{z z}\right) \propto(0,1,0),(b)\left(\sigma_{x x}, \sigma_{y y}, \sigma_{z z}\right) \propto(0,0,1),(c)\left(\sigma_{x x}, \sigma_{y y}, \sigma_{z z}\right) \propto$ $(1,1,1),(d)\left(\sigma_{x x}, \sigma_{y y}, \sigma_{z z}\right) \propto(1,1,0),(e)\left(\sigma_{x x}, \sigma_{y y}, \sigma_{z z}\right) \propto(0,1,1)$ and $(f)\left(\sigma_{x x}, \sigma_{y y}, \sigma_{z z}\right)=(0,0,0)$

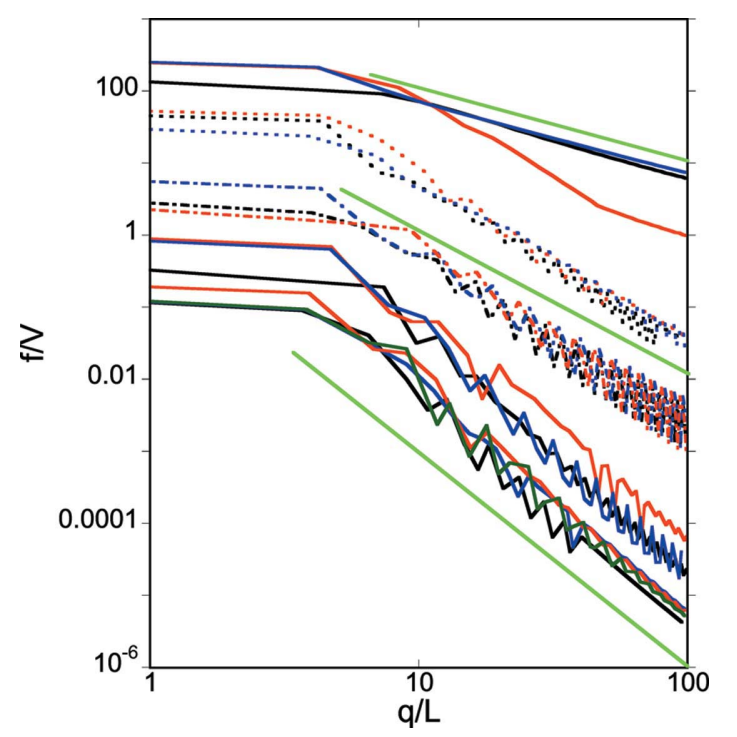

Figure 2

Dependence of the amplitude form factor maxima on $q$ for different orientations, for $\Delta \phi=2 \pi$ and for $\left(\sigma_{x x}, \sigma_{y y}, \sigma_{z z}\right) \propto(0,1,0)$. The form factor amplitude, $f$, is normalized by the cube volume, $V$. The $\mathbf{Q}$ direction is (001). The shear stresses are null. The curves corresponding to different orientations are grouped and shifted by a multiplicative factor $M: \mathbf{q}$ normal to faces, $M=1000$, full lines: black: (001) direction, red: (010) direction, blue: (100) direction; q perpendicular to $\langle 100\rangle$ edges, $M=$ $1000^{3 / 4}$, dotted lines: black: (011) direction, red: (021) direction, blue: (012) direction; q perpendicular to $\langle 010\rangle$ edges, $M=1000^{1 / 2}$, dashed lines: black: (101) direction, red: (201) direction, blue: (102) direction; q perpendicular to $\langle 001\rangle$ edges, $M=1000^{1 / 4}$, full lines: black: (110) direction, red: (210) direction, blue: (120) direction; $\mathbf{q}$ in a generic direction, $M=1$, full lines: black: (111) direction, red: (112) direction, blue: (121) direction; green: (211) direction. The three light-green segments correspond to $q^{-1}$, $q^{-2}$ and $q^{-3}$ curves. The asymptotic behaviours of each direction group normal to a face, perpendicular to an edge and generic direction correspond to a different $q$ exponent, $-1,-2$ and -3 . 
2004) and, therefore, we expect $\Delta \phi<2 \pi$ for pseudomorphic inclusions or pseudomorphic huts, i.e. inclusions on a surface (Dujardin et al., 2006). Lastly, it is worth noting that a value around $2 \pi$ was found by Robinson and co-workers in their seminal work on coherent scattering by a $\mathrm{Pb}$ nanocrystal (Pfeifer et al., 2006).

For an undeformed cube of size $L$, the dependence of the form factor asymptotic behaviour on $q$ orientation leads to a quite observable contrast in the form factor landscape for $q L=$ 20. Fig. 1 exhibits such a dependence of $f$ on the $\mathbf{q}$ orientation for $\Delta \phi=2 \pi, \mathbf{Q} / /(001)$ and various orientations of the stress $\bar{\sigma}_{0}$. The main characteristics of the landscape are kept: highintensity poles which correspond to directions normal to a face, high intensity on great circles joining these poles which correspond to directions perpendicular to an edge, weak intensity in generic directions. Nevertheless, some features affect this general behaviour: (i) for $\left(\sigma_{x x}, \sigma_{y y}, \sigma_{z z}\right) \propto(0,1,0)$ or $\left(\sigma_{x x}, \sigma_{y y}, \sigma_{z z}\right) \propto(1,1,0)$, the intensity overload is kept around the (010) pole but disappears in the exact pole position; the same behaviour occurs for the great circle normal to the edges $\langle 001\rangle$; (ii) for $\left(\sigma_{x x}, \sigma_{y y}, \sigma_{z z}\right) \propto(0,0,1),\left(\sigma_{x x}, \sigma_{y y}, \sigma_{z z}\right)$ $\propto(1,1,1)$ and $\left(\sigma_{x x}, \sigma_{y y}, \sigma_{z z}\right) \propto(0,1,1)$, this great circle normal to the edges $\langle 001\rangle$ is almost invisible. As for the case of undeformed polyhedra, the landscape is closely linked to the

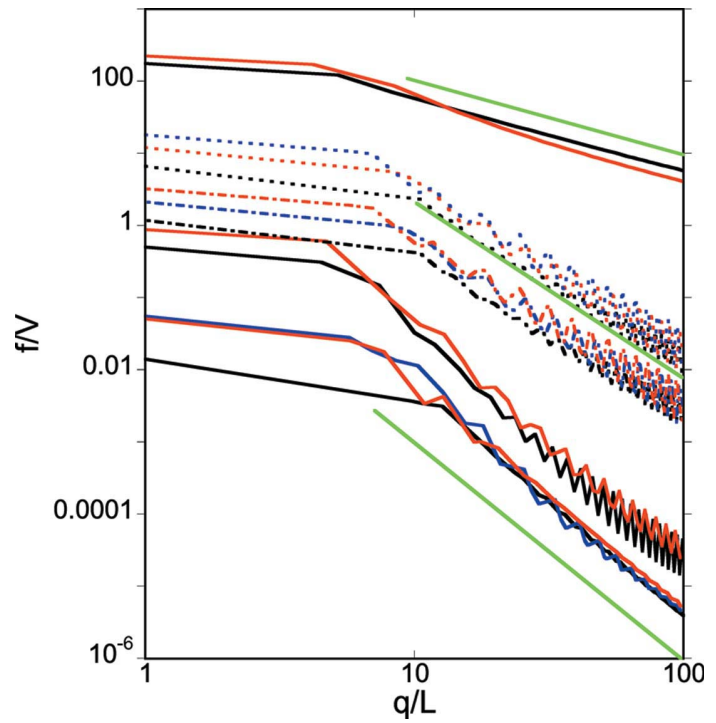

Figure 3

Dependence of the amplitude form factor maxima on $q$ for different orientations, for $\Delta \phi=2 \pi$ and for $\left(\sigma_{x x}, \sigma_{y y}, \sigma_{z z}\right) \propto(0,0,1)$. The form factor amplitude, $f$, is normalized by the cube volume, $V$. The $\mathbf{Q}$ direction is (001). The shear stresses are null. The curves corresponding to different orientations are grouped and shifted by a multiplicative factor $M$ : $\mathbf{q}$ normal to faces, $M=1000$, full lines: black: (001) direction, red: (010) direction and (100) direction; q perpendicular to $\langle 100\rangle$ edges, $M=1000^{3 / 4}$, dotted lines: black: (011) direction, red: (021) direction, blue: (012) direction; q perpendicular to $\langle 010\rangle$ edges, $M=1000^{1 / 2}$, dashed lines: black: (101) direction, red: (201) direction, blue: (102) direction; q perpendicular to $\langle 001\rangle$ edges, $M=1000^{1 / 4}$, full lines: black: (110) direction, red: (210) direction and (120) direction; $\mathbf{q}$ in a generic direction, $M=1$, full lines: black: (111) direction, red: (112) direction, blue: (121) direction and (211) direction. The three light-green segments correspond to $q^{-1}, q^{-2}$ and $q^{-3}$ curves. The asymptotic behaviours of each direction group - normal to a face, perpendicular to an edge and generic direction - correspond to a different $q$ exponent, $-1,-2$ and -3 .

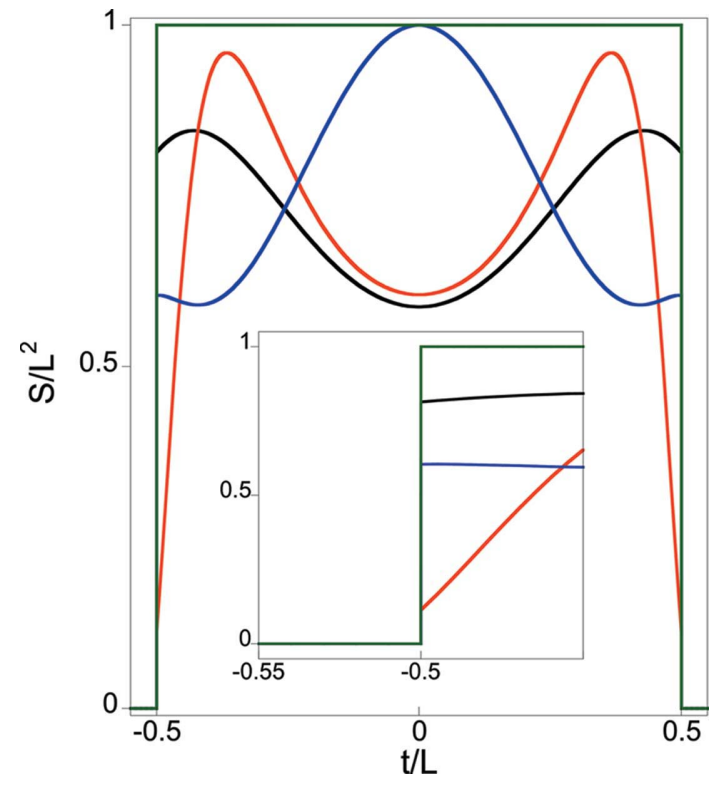

Figure 4

Dependence of the section area modulus, $S$, on the section position, $t$, for different $\{100\}$ orientations and for $\Delta \phi=2 \pi$. The position is normalized by $L$, the cube size, and the section is normalized by $L^{2}$. The $\mathbf{Q}$ direction is (001). The shear stresses are null. $\left(\sigma_{x x}, \sigma_{y y}, \sigma_{z z}\right) \propto(0,1,0)$. Black: section normal to the (100) direction, red: section normal to the (010) direction, blue: section normal to the (001) direction, green: section normal to any of the $\{100\}$ directions in the absence of strain. The inset is an enlarged view of the main discontinuity.

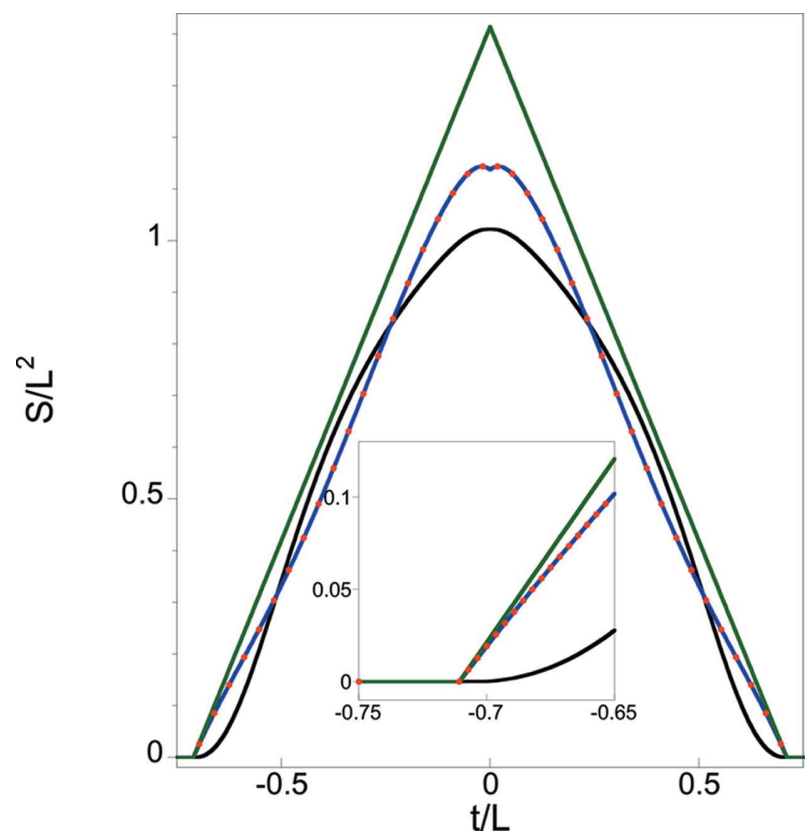

Figure 5

Dependence of the section area modulus, $S$, on the section position, $t$, for different $\{110\}$ orientations and for $\Delta \phi=2 \pi$. The position is normalized by $L$, the cube size, and the section is normalized by $L^{2}$. The $\mathbf{Q}$ direction is (001). The shear stresses are null. $\left(\sigma_{x x}, \sigma_{y y}, \sigma_{z z}\right) \propto(0,0,1)$. Black: section normal to the (110) direction, red: section normal to the (101) direction, blue: section normal to the (011) direction, green: section normal to any of the $\{110\}$ directions in the absence of strain. The inset is an enlarged view of the main discontinuity. 


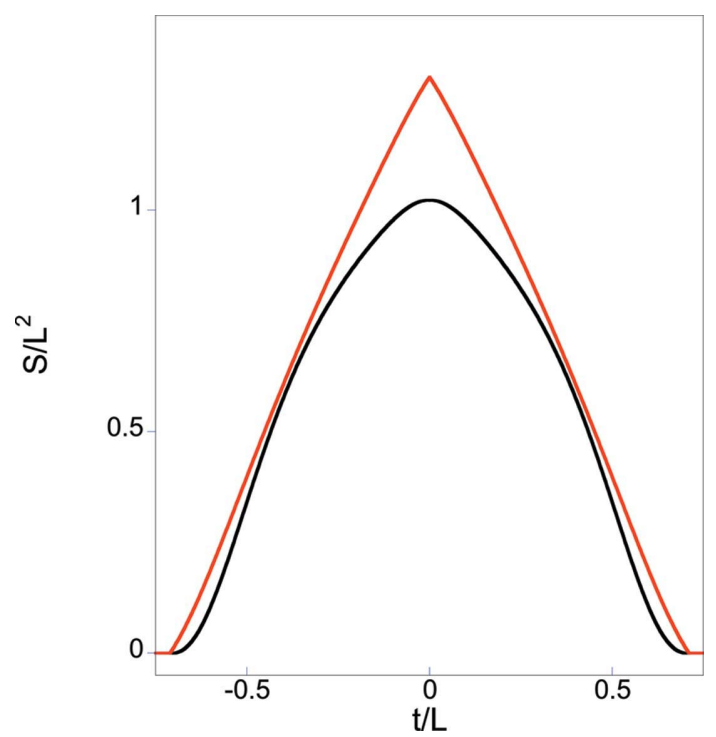

Figure 6

Dependence of the section area modulus, $S$, on the section position, $t$, for a (110) direction. The position is normalized by $L$, the cube size, and the section is normalized by $L^{2}$. The $\mathbf{Q}$ direction is (001). The shear stresses are null. $\left(\sigma_{x x}, \sigma_{y y}, \sigma_{z z}\right) \propto(0,0,1)$. Black: density phase amplitude equal to $2 \pi$, red: density phase amplitude equal to $\pi$.

asymptotic behaviour of $f(q)$. Figs. 2 and 3 illustrate this point. The observable and undisturbed poles are associated with $1 / q$ asymptotic behaviour, the observable and undisturbed great circles are associated with $1 / q^{2}$ asymptotic behaviour, and the generic directions correspond to $1 / q^{3}$ asymptotic behaviour. In contrast, perturbation or suppression of landscape features corresponds to an increase of the coefficient of the $1 / q^{n}$ asymptotic behaviour: this is clearly visible for the (010) pole in Fig. 2 and for directions normal to the edges $\langle 001\rangle$ in both Figs. 2 and 3. The generalization of the sections method developed by Croset (2018) allows us to understand these results. By choosing as Cartesian coordinate system, $(X, Y, Z)$, a system having its $Z$ axis parallel to $\mathbf{q}, f$ can be written

$$
\begin{aligned}
f(\mathbf{q}) & =\int\left\{\iint_{\mathscr{S}(t)} \exp \left[-i \phi\left(r ; \mathbf{Q}, \sigma_{0}\right)\right] \mathrm{d} X \mathrm{~d} \mathrm{Y}\right\} \exp (-i q t) \mathrm{d} t \\
& =\int A_{\mathrm{c}}(t) \exp (-i q t) \mathrm{d} t,
\end{aligned}
$$

where $\mathscr{S}(t)$ is the section of the crystal by the plane normal to q at $Z=t$ and $A_{\mathrm{c}}(t)$ is a complex area given by the integral of the complex density on the section $\mathscr{S}(t)$ given by

$$
A_{\mathrm{c}}(t)=\iint_{\mathscr{Y}(t)} \exp \left[-i \phi\left(r ; \mathbf{Q}, \sigma_{0}\right)\right] \mathrm{d} X \mathrm{~d} Y .
$$

Because of the triangular inequality, we have

$$
\left|A_{\mathrm{c}}(t)\right|<\iint_{\mathscr{S}(t)} \mathrm{d} X \mathrm{~d} Y=A(t),
$$

where $A(t)$ is the real area of the section $\mathscr{S}(t)$. This result indicates that the discontinuities of $\left|A_{\mathrm{c}}(t)\right|$ associated with the entry and the exit of the crystal are weaker for the deformed crystal when compared with the undeformed crystal case. This is particularly clear in Fig. 4: in the (010) direction, the entry discontinuity is a Heaviside step for the undeformed crystal while it is essentially a slope discontinuity for the deformed crystal, the Heaviside step only being visible at low scale. In the same way, in the (110) direction, the slope discontinuity is replaced with a curvature discontinuity as shown in Fig. 5. (c)

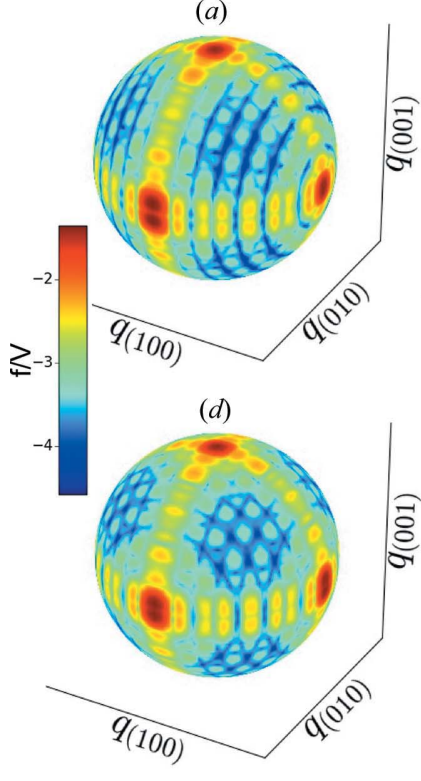

(b)

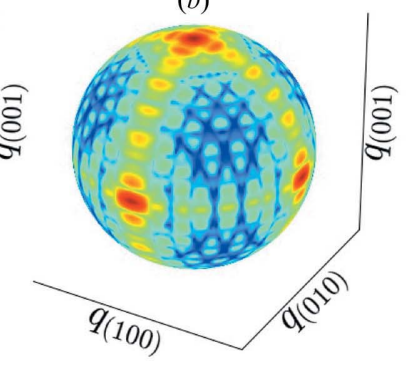

$(e)$

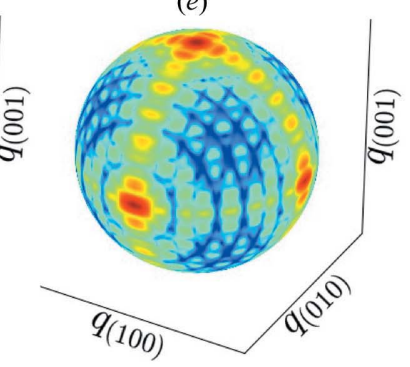

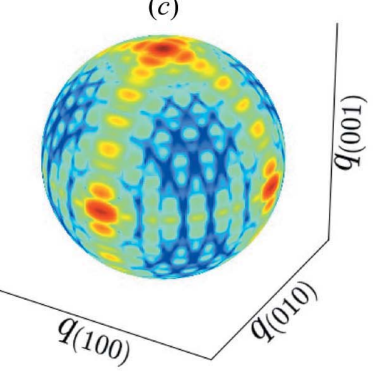

$(f)$

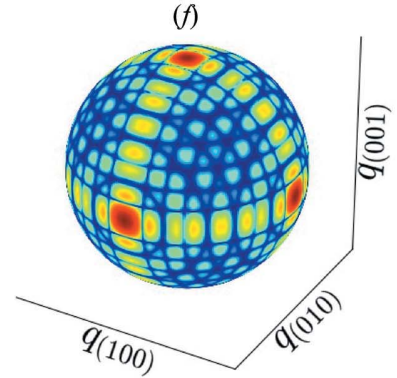

Figure 7

Orientation dependence of the amplitude form factor of a cubic inclusion of size $L$ for $q L=20$ and for $\Delta \phi=\pi$. The colour scale is logarithmic and common to all the images. The form factor amplitude, $f$, is normalized by the cube volume, $V$. The $\mathbf{Q}$ direction is (001). The shear stresses are null for all the images. $(a)\left(\sigma_{x x}, \sigma_{y y}, \sigma_{z z}\right) \propto(0,1,0),(b)\left(\sigma_{x x}, \sigma_{y y}, \sigma_{z z}\right) \propto(0,0,1),(c)\left(\sigma_{x x}, \sigma_{y y}, \sigma_{z z}\right) \propto$ $(1,1,1),(d)\left(\sigma_{x x}, \sigma_{y y}, \sigma_{z z}\right) \propto(1,1,0),(e)\left(\sigma_{x x}, \sigma_{y y}, \sigma_{z z}\right) \propto(0,1,1)$ and $(f)\left(\sigma_{x x}, \sigma_{y y}, \sigma_{z z}\right) \propto(0,0,0)$.
The sections method allows the study of the crucial role of $\Delta \phi$. Fig. 6 shows that a decrease from $2 \pi$ to $\pi$ for $\Delta \phi$ restores the slope discontinuity for the section in the (110) direction. As clearly shown in Fig. 7, all the features of the form factor landscape associated with the undeformed crystal are visible for a deformed crystal with $\Delta \phi=\pi$ : poles in directions normal to the faces, great circles corresponding to directions perpendicular to the edges. To understand these results, a detailed study of the displacement field is useful. Let us take as an example the case $\mathbf{Q} / /(001)$, $\left(\sigma_{x x}, \sigma_{y y}, \sigma_{z z}\right) \propto(0,0,1)$ and $\mathbf{q} / /(110)$. For $-L /(2)^{1 / 2}<t<L /(2)^{1 / 2}$, the section, $\mathscr{S}(t)$, is a rectangle with a constant edge length in the direction [001] and a variable edge length in the direction

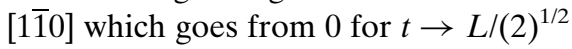
to $L(2)^{1 / 2}$ for $t=0$. Fig. 8 shows the density phase landscape for various values of $t$. Since $\mathbf{Q}$ is parallel to (001), 


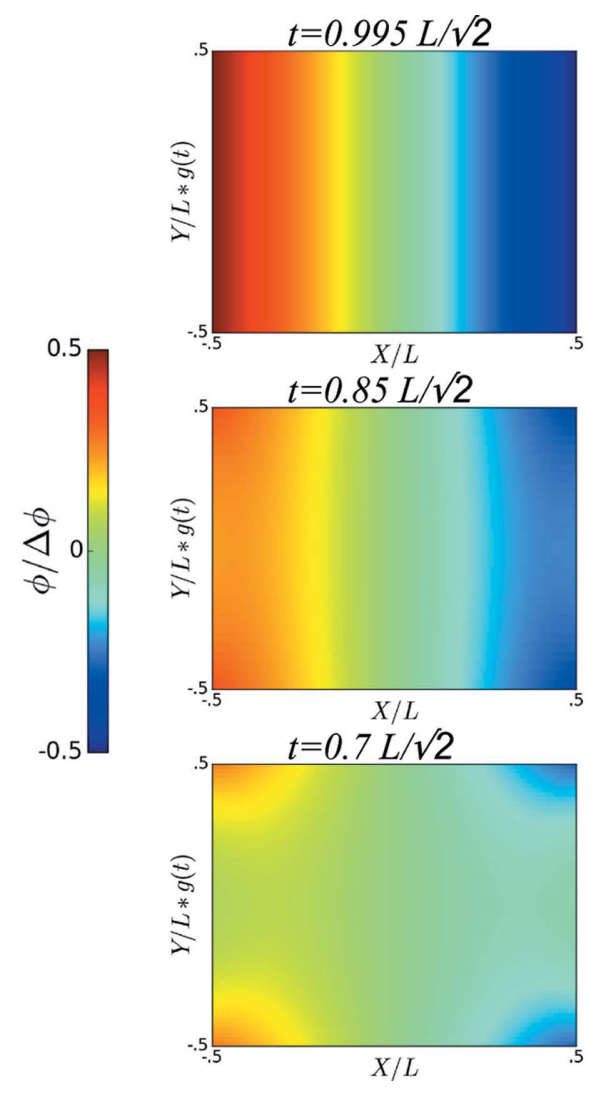

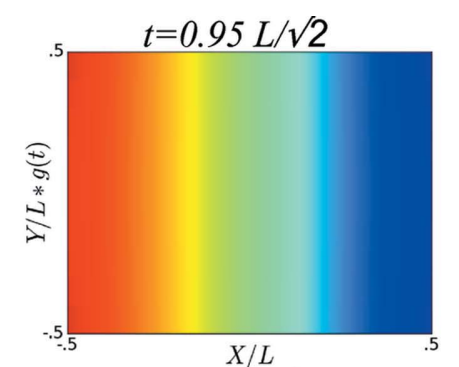
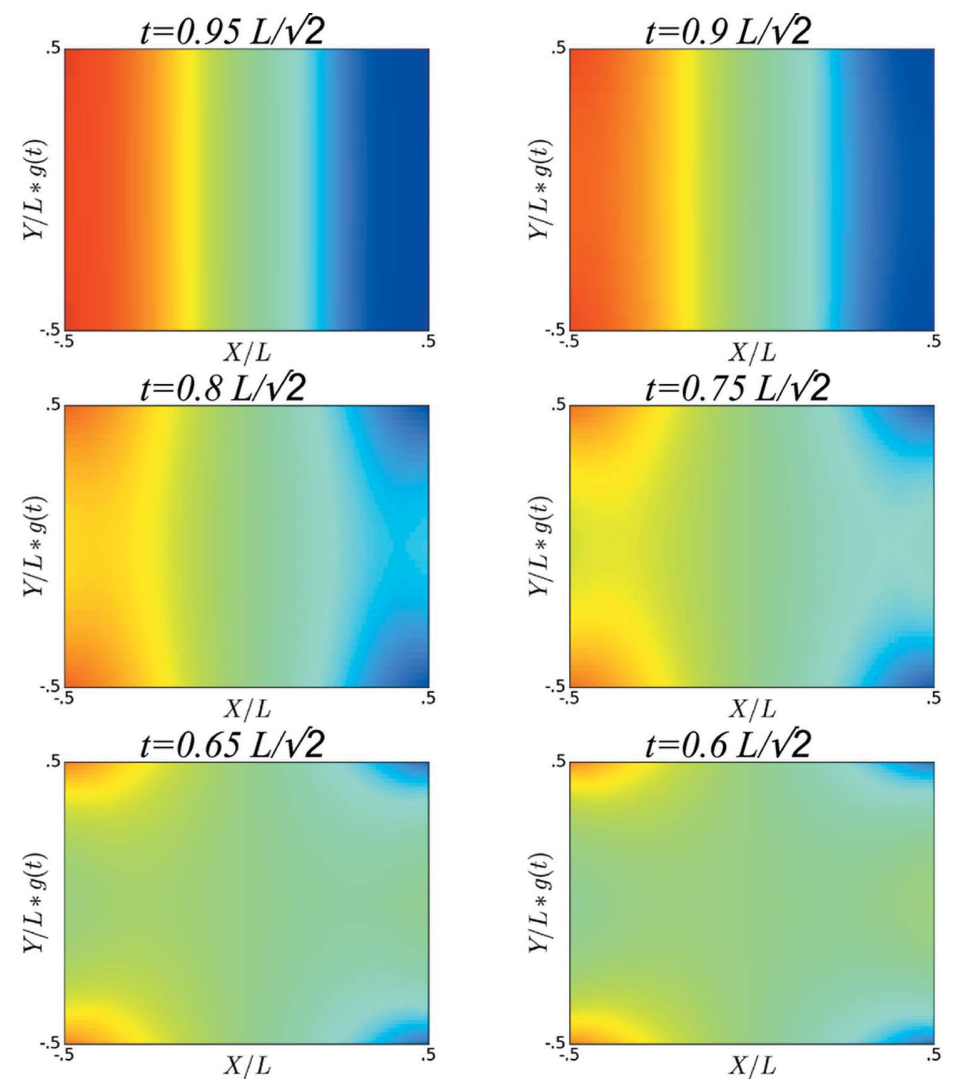

Figure 8

Variation of the density phase in the section $\mathscr{S}(t)$ for various values of $t$. The $\mathbf{Q}$ direction is (001) and the $\mathbf{q}$ direction is (110). The shear stresses are null for all the images and $\left(\sigma_{x x}, \sigma_{y y}, \sigma_{z z}\right) \propto(0,0,1)$. The colour scale is linear and is the same for all the sections. The varying side of the section is normalized to 1 by the function $g(t)=1 /\left(2-4 t^{2} / L^{2}\right)^{1 / 2}$.

the density phase landscape is identical to the landscape of $u_{x}$, the $x$ component of the displacement. Several features can be seen:

(i) We have $\phi(-X)=-\phi(X)$ and $\phi(-Y)=\phi(Y)$.

(ii) The amplitude of the phase variation increases with $t$ and reaches $\Delta \phi$ for $t \rightarrow L /(2)^{1 / 2}$.

(iii) The phase variation with $Y$ is almost null for high $t$ while it presents oscillation for low $t$.

For $t \rightarrow L /(2)^{1 / 2}$ and $\Delta \phi=2 \pi, \phi$ varies consequently from $-\pi$ to $+\pi$ from the left side to the right side of the section. The integral of the first harmonic of the complex density is therefore null for $t \rightarrow L /(2)^{1 / 2}$. This effect overlaps with the variation of the real area of the section. This explains the substitution of a parabolic shape for a linear shape for the variation of the area modulus in Fig. 6.

Most of the properties of the density phase landscape shown in Fig. 8 are generic and are not due to our special choice of an Eshelby inclusion. There are two reasons for this:

First, since the $X$ axis coincides with the $z$ axis, the change in sign of the displacement when passing from the left side of the section to the right side of the section is due to the fact that on the crystal boundaries the displacements are parallel to the applied forces. This behaviour is generic since it is due to the $1 / r$ behaviour of the Green function highlighted in the previous section.
Second, the increase in the displacement when the section is near an edge which is the case for $t \rightarrow L /(2)^{1 / 2}$ is due to a tip effect. This effect, similar to the tip effect in electrostatics, is generic in cases of linear elasticity and is a consequence of the constitutive equations being quite similar to the electrostatics ones [see for example Fig. 13 in Xiong et al. (2014)].

These two remarks are of major importance: whereas for more complex situations than the pseudomorphic inclusion the phase landscape of the sections would be less simple, the behaviour of the first harmonic of the complex density will be kept. As a result, for $\Delta \phi \sim 2 \pi$ the singularities of the complex area modulus occurring at the crystal boundaries will be similar to those obtained for our example. This generality can be compared with the generality obtained in previous work on the observation of elastic displacement modes by grazingincidence X-ray diffraction (Prévot et al., 2007).

\section{Conclusion}

Taking, for example, the pseudomorphic inclusion for which the displacement field can be calculated analytically, we have been able to study the influence of the elastic relaxations on the form factor of cubic nanocrystals. We have highlighted the role of a control parameter, $\Delta \phi$, the total amplitude of the variation of the complex density phase. The asymptotic 
behaviours of the form factor of undeformed crystals are preserved for $\Delta \phi<2 \pi$ while they are modified in some directions when $\Delta \phi \geq 2 \pi$. An a priori guess of the orientation of the stress allows us to determine the scattering directions for which the asymptotic behaviours associated with the crystal shape are preserved and the scattering directions for which they are strongly modified. Using the sections method we showed that these results are generic and not due to the simplicity of our example. Thanks to the development of $\mathrm{X}$-ray free-electron laser facilities, coherent scattering studies of the shape and the deformations of nanocrystals will become easier in the near future (Vartanyants et al., 2007). Our results may help to define a measuring strategy when performing such studies.

\section{Acknowledgements}

This work found its motivation in conversations with Vincent Favre-Nicolin and Olivier Thomas. We thank both of them and are indebted to Romain Bernard for careful reading.

\section{References}

Chamard, V., Allain, M., Godard, P., Talneau, A., Patriarche, G. \& Burghammer, M. (2015). Sci. Rep. 5, 9827.

Croset, B. (2017). J. Appl. Cryst. 50, 1245-1255.

Croset, B. (2018). J. Appl. Cryst. 51, 1005-1012.
Diaz, A., Chamard, V., Mocuta, C., Magalhães-Paniago, R., Stangl, J., Carbone, D., Metzger, T. H. \& Bauer, G. (2010). New J. Phys. 12, 035006.

Dujardin, R., Poydenot, V., Schülli, T. U., Renaud, G., Ulrich, O., Barski, A., Derivaz, M., Colonna, S. \& Metzger, T. (2006). J. Appl. Phys. 99, 063510.

Eshelby, J. D. (1957). Proc. R. Soc. A, 241, 376-396.

Favre-Nicolin, V., Mastropietro, F., Eymery, J., Camacho, D., Niquet, Y. M., Borg, B. M., Messing, M. E., Wernersson, L.-E., Algra, R. E., Bakkers, E. P. A. M., Metzger, T. H., Harder, R. \& Robinson, I. K. (2018). New J. Phys. 12, 035013.

Hill, M. O., Calvo-Almazan, I., Allain, M., Holt, M. V., Ulvestad, A., Treu, J., Koblmüller, G., Huang, C., Huang, X., Yan, H., Nazaretski, E., Chu, Y. S., Stephenson, G. B., Chamard, V., Lauhon, L. J. \& Hruszkewycz, S. O. (2018). Nano Lett. 18, 811-819.

Hruszkewycz, S. O., Allain, M., Holt, M. V., Murray, C. E., Holt, J. R., Fuoss, P. H. \& Chamard, V. (2017). Nat. Mater. 16, 244-251.

Lifshitz, F. M. \& Landau, L. D. (1995). Theory of Elasticity. Oxford: Butterworth-Heinenman.

Pfeifer, M. A., Williams, J. W., Vartanyants, I. A., Harder, R. \& Robinson, I. K. (2006). Nature, 442, 63-66.

Prévot, G., Coati, A., Croset, B. \& Garreau, Y. (2007). J. Appl. Cryst. 40, 874-882.

Shin, J., Cornelius, T. W., Labat, S., Lauraux, F., Richard, M.-I., Richter, G., Blanchard, N. P., Gianola, D. S. \& Thomas, O. (2018). J. Appl. Cryst. 51, 781-788.

Stroh, A. N. (1958). Philos. Mag. 3, 625-646.

Ting, T. C. T. (2004). Q. J. Mech. Appl. Math. 45, 119-139.

Vartanyants, I. A., Robinson, I. K., McNulty, I., David, C., Wochner, P. \& Tschentscher, Th. (2007). J. Synchrotron Rad. 14, 453-470.

Xiong, G., Moutanabbir, O., Reiche, M., Harder, R. \& Robinson, I. (2014). Adv. Mater. 26, 7747-7763. 